BEHAVIORAL AND BRAIN SCIENCES (2008) 31, 599-60.

\title{
Anticipation is the key to understanding music and the effects of music on emotion
}

doi:10.1017/S0140525X08005542

Peter Vuust ${ }^{\mathrm{a}, \mathrm{b}}$ and Chris D. Frith ${ }^{\mathrm{a}, \mathrm{c}}$

${ }^{a}$ Center of Functionally Integrative Neuroscience, Aarhus University Hospital, 8000

Aarhus C, Denmark; bRoyal Academy of Music, 8000 Aarhus, Denmark; 'Wellcome

Trust Centre for Neuroimaging, University College, London, WC1 N 3BG, United

Kingdom.

pv@pet.auh.dk http://www.musik-kons.dk/foku/pvuust.php

skaacfr@ucl.ac.uk http://www.interacting-minds.net

Abstract: There is certainly a need for a framework to guide the study of the physiological mechanisms underlying the experience of music and the emotions that music evokes. However, this framework should be organised hierarchically, with musical anticipation as its fundamental mechanism.

Juslin \& Västfjäll (J\&V) claim that the study of musical emotions has suffered from a neglect of the underlying psychological mechanisms evoking these emotions and propose that these mechanisms can be summarized as (a) brain stem reflexes, (b) evaluative conditioning, (c) emotional contagion, (d) visual imagery, (e) episodic memory, and (f) musical expectancy. A problem with these categories is that they are not ordered hierarchically, are not mutually exclusive, and only category (f) - musical expectancy - directly links musical and psychological mechanisms as such. This limits the scope of the proposed framework somewhat, especially if its purpose is to act as a guideline for experiments trying to identify the modularity for the brain structures involved in the processing of musical emotions. We believe that the framework would be more useful if the mechanisms for evoking musical emotions were organized hierarchically such that musical expectancy was seen as the most fundamental mechanism underlying the other mechanisms.

It is hard to imagine that musical emotions are evoked without some sort of musical meaning assigned to what is heard, unless we think of emotions, such as fear, evoked by the mere advent of a sudden loud, scary sound. However, in such a case, it is questionable whether one would define this as music. Most music theoreticians (Cooper \& Meyer 1960; Lerdahl \& Jackendoff 1977; 1999; Meyer 1956; Monelle 1992) consider musical anticipation as one of the principal means by which music conveys meaning and emotion. According to this point of view, understanding music (Cooper \& Meyer 1960; Lerdahl 1971; Lerdahl \& Jackendoff 1999, pp. 145-60; Meyer 1956; Monelle 1992) is related to the anticipatory interplay between local auditory events and a deeper structural layer partly inherent in the music itself, and partly provided by mental structures in the listeners that is induced by the music (Palmer \& Krumhansl 1990; Vuust et al. 2006a). In short, the musical experience is dependent on the structures of the actual music, as well as on the expectations of the interpreting brain. These expectations are dependent on long-term learning of musical 
structures (culture-dependent statistical learning), familiarity with a particular piece of music, and short-term memory for the immediate musical history while listening to a musical piece, as well as on deliberate listening strategies (Huron 2006; Vuust et al. 2006b). Brain structures underlying musical expectation are thus shaped by culture, as well as by personal listening history and musical training (Vuust et al. 2005). Moreover, as soon as one hears the first sound of a musical piece, anticipational structures enabling anticipation, such as meter, tonality, and memory for particular musical pieces, seem to be in place already and therefore unavoidable (e.g., see Brochard et al. 2003). Thus, it is difficult to imagine any of the proposed mechanisms acting without the involvement of musical expectation.

Judging from their Table 4, J\&V believe that musical expectation is something that develops slowly over time during listening experience and is not fully developed until the ages of 5 to 11 years. This may well be correct if musical expectation is restricted to anticipation of complex musical structures, such as the hierarchy of harmony dependent on long-term learning (see, e.g., Leino et al. 2007). However, expectation of the more simple repetitive sound patterns, such as pitch deviants in successive pitch trains, which is a part of all music, has been detected even before birth, as indicated by the mismatch negativity (MMN) measured by electroencephalography (EEG) or magnetoencephalography (MEG) (Huotilainen et al. 2005). Moreover, in an elegant study, Winkler et al. (1996) showed that the auditory predictive model is updated for each new acoustic event in the sound environment, indicating that the anticipatory structures of music are in constant flux during the listening experience. These results demonstrate that anticipation has a role at many levels in the hierarchy of musical structure.

Furthermore, J\&V also claim that the degree of volitional influence on musical anticipation is low. However, we recently conducted a study in which musicians were asked to maintain either the main meter or a countermeter while listening to Sting's "The Lazarus Heart" (Vuust et al. 2006b). In this experiment, the subjects could volitionally impose two very different anticipatory frameworks onto the music. Deliberately listening to a melody from the perspective of two different tonalities would be another example of volitional control of the anticipatory framework.

The relationship between musical expectancy and emotion was originally explored by Meyer (1956) and has recently been elaborated upon convincingly by Huron (2006) in his book Sweet Anticipation. If we consider music expectation/anticipation as the fundamental mechanism for the musical experience, then this maps nicely onto recent theories of how the brain works. Karl Friston (2005) has provided a promising model of brain function, in which predictive coding, as a central principle of brain function, provides an account of how the brain identifies and categorizes the causes of its sensory inputs (for similar viewpoints, see Shepard 2001; Tononi \& Edelman 1998). The model posits a hierarchical organization whereby lower-level brain regions estimate predictions of their expected input based on contextual information through backwards connections from higher-level regions. A comparison between prediction and actual input produces an error term that, if sufficiently large, will be fed back to call for an update of the model. This generates a recursive process, which aims at minimizing the difference between input and prediction. As the 
representational capacity of any neuronal assembly in this model is dynamic and context sensitive, this, among other issues, addresses the problem of top-down control (Frith \& Dolan 1997; Roepstorff \& Frith 2004). Lately, we have argued that processing violations of musical anticipation in different aspects of the music (e.g., rhythm/harmony) evokes different error messages (MMN/ early anterior negativity [EAN]) and networks (Vuust et al., in press). These effects are training dependent and can be explained by the predictive coding theory. Thus, in our opinion, musical expectation is a good candidate for the fundamental mechanism guiding the experience of musical meaning as well as emotion. Anticipation in itself may evoke a wealth of emotions, such as awe, surprise, discomfort, the sensation of swing, and so on. According to Huron (2006), this is due to a variety of different survival-related responses to anticipation, in particular the "prediction response" that rewards fulfilled expectations.

However, anticipatory structures such as meter and tonality act indirectly on the other proposed mechanisms, in that they form the basis for musical memory, as well as for musical meaning.

If we consider the vast amount of neuroscientific research on music that has been published in recent years, it is certainly true that the studies of musical emotions seem to be pointing in different directions. Consider, for instance, the very different activation patterns reported in studies of major and minor mode music (Green et al. 2008; Khalfa et al. 2005; Pallesen et al. 2005) supposedly evoking very simple emotions (happy/sad). Even though these results may be due to many different factors contributing to the emotional state of the subjects under different experimental conditions, we agree with the J\&V that one of the reasons for these contradictory results may be the lack of a theoretical framework. However, this framework needs to be organized hierarchically with music anticipation as the guiding mechanism

Cooper, G. W. \& Meyer, L. B. (1960) The rhythmic structure of music. University of Chicago Press.

Friston, K. (2005) A theory of cortical responses. Philosophical Transactions of the Royal Society of London B 360:815 - 56.

Frith, C. \& Dolan, R. J. (1997) Brain mechanisms associated with top-down processes in perception. Philosophical Transactions of the Royal Society of London, B: Biological Sciences 352:1221 - 30.

Green, A. C., Bæhrentsen, K. B., Stødkilde-Jørgensen, H. Wallentin, M.,Roepstorff, A. \& Vuust, P. (2008) Music in minor activates limbic structures: A relation to dissonance? NeuroReport 19:711 -15.

Huotilainen, M., Kujala, A., Hotakainen, M., Parkkonen, L., Taulu, S., Simola, J.,Nenonen, J., Karjalainen, M. \& Naatanen, R. (2005) Short-term memory functions of the human fetus recorded with magnetoencephalography. NeuroReport 16:81 - 84.

Khalfa, S., Schon, D., Anton, J. L. \& Liegeois-Chauvel, C. (2005) Brain regions involved in the recognition of happiness and sadness in music. NeuroReport 16:1981 -84.

Leino, S., Brattico, E., Tervaniemi, M. \& Vuust, P. (2007) Representation of harmony rules in the human brain: Further evidence from event-related potentials. Brain Research 1142:169 - 77. 
Lerdahl, F. \& Jackendoff, R. (1977) Toward a formal theory of tonal music. Journal of Music Theory 21(1):111- 71.

Lerdahl, F. \& Jackendoff, R. (1999) A generative theory of music. MIT Press. Monelle, R. (1992) Linguistics and semiotics in music. Harwood Academic.

Pallesen, K. J., Brattico, E., Bailey, C., Korvenoja, A., Koivisto, J., Gjedde, A. \& Carlson, S. (2005) Emotion processing of major, minor, and dissonant chords: A functional magnetic resonance imaging study. Annals of the New York Academy of Sciences 1060:450 - 53.

Palmer, C. \& Krumhansl, C. L. (1990) Mental representations for musical meter. Journal of Experimental Psychology: Human Perception and Performance 16:728 - 41.

Roepstorff, A. \& Frith, C. (2004) What's at the top in the top-down control of action? Script-sharing and "top-top" control of action in cognitive experiments. Psychological Research 68:189 - 98

Shepard, R. N. (2001) Perceptual-cognitive universals as reflections of the world. Behavioral and Brain Sciences 24:581 - 601.

Tononi, G. \& Edelman, G. M. (1998) Consciousness and the integration of information in the brain. Advances in Neurology 77:245 - 79.

Vuust, P., Østergaard, L., Pallesen, K. J., Bailey, C. \& Roepstorff, A. (in press) Predictive coding of music. Cortex

Vuust, P., Østergaard, L. \& Roepstorff, A. (2006a) Polyrhythmic communicational devices appear as language in the brains of musicians. In: ICMPC9 Proceedings of the International Conference on Music Perception and Cognition, ESCOM, Bologna, 2006, pp. 1159 - 67. (Unpublished Proceedings.)

Vuust, P., Pallesen, K. J., Bailey, C., Van Zuijen, T. L., Gjedde, A., Roepstorff, A. \& Østergaard, L. (2005) To musicians, the message is in the meter: Pre-attentive neuronal responses to incongruent rhythm are left-lateralized in musicians. NeuroImage 24:560 - 64 .

Vuust, P., Roepstorff, A., Wallentin, M., Mouridsen, K. \& Østergaard, L. (2006b) It don't mean a thing ... Keeping the rhythm during polyrhythmic tension, activates language areas (BA47). NeuroImage 31:832 -41.

Winkler, I., Karmos, G. \& Naatanen, R. (1996) Adaptive modeling of the unattended acoustic environment reflected in the mismatch negativity eventrelated potential. Brain Research 742:239 -52. 